\title{
Kernos
}

Revue internationale et pluridisciplinaire de religion grecque antique

16 | 2003

Varia

\section{P.F. BEATRICE, Anonymi Monophysitae Theosoophia}

\section{Peter Van Deun}

\section{(2) OpenEdition}

\section{Journals}

Édition électronique

URL : http://journals.openedition.org/kernos/857

DOI : $10.4000 /$ kernos.857

ISSN : 2034-7871

Éditeur

Centre international d'étude de la religion grecque antique

Édition imprimée

Date de publication : 1 janvier 2003

Pagination : 387-389

ISSN : 0776-3824

Référence électronique

Peter Van Deun, «P.F. BEAtrice, Anonymi Monophysitae Theosoophia », Kernos [En ligne], 16 | 2003, mis en ligne le 14 avril 2011, consulté le 24 septembre 2020. URL : http://journals.openedition.org/kernos/ 857 ; DOI : https://doi.org/10.4000/kernos.857 
lité est synonyme de mythe et témoigne d'une Présence) qui demande au lecteur une ténacité certaine pour la suivre dans tous les détails.

Véronique Dasen

(Université de Fribourg)

Pier Franco BeAtrice, Anonymi Monophysitae Theosophia. An Attempt at Reconstruction, Leiden, Brill, 2001, LXXI + 140 p. (Suppl. to Vigiliae Christianae, 56). ISBN : 90-04-11798-9.

En 1995, Hartmut Erbse a fait paraître un livre intitulé Theosophorum Graecorum Fragmenta (Bibliotbeca Scriptorum Graecorum et Romanorum Teubneriana), Stuttgart/ Leipzig (dorénavant Erbse, Fragmenta); il s'agit de l'édition critique de différentes recensions de textes 'théosophiques' grecs, tant païens que chrétiens (sentences, oracles, apophtegmes et d'autres fragments); cette édition revoit celle que le même auteur a établie dans ses Fragmente griechischer Theosopbien (Hamburger Arbeiten zur Altertumswissenschaft 4), Hamburg, 1941 (dorénavant Erbse, Fragmente). À peine cinq années sont passées, et voici une nouvelle édition, préparée par Pier Franco Beatrice. La première remarque qui s'impose ici, est la suivante : encore une édition du même texte! Il n'y a donc rien d'étonnant à ce que Beatrice consacre une partie de son introduction à montrer l'importance de son travail. Tandis que Erbse a juxtaposé les différentes recensions et les multiples fragments, Beatrice essaie de reconstruire le texte original de l'œuvre appelée $\Theta \epsilon о \sigma o \phi l a$ et divisée en quatre livres; les résultats de reconstruction auxquels Beatrice a abouti, nous semblent convaincants. Probante est également la datation et l'attribution du texte qu'il propose: il l'attribue à Sévère, patriarche monophysite d'Antioche de 512 à 518; donc l'ouvrage revient au début du $\mathrm{vI}^{\mathrm{e}}$ siècle et s'inspire de l'hérésie monophysite. Une brève étude de la tradition manuscrite et des éditions imprimées est suivie du texte critique de l'œuvre; un index locorum Sacrae Scripturae et un index fontium in textu laudatorum facilitent la consultation du livre (malheureusement il n'y a ni index des incipit des fragments, ni index graecitatis).

Nous voudrions formuler deux remarques générales sur le texte établi. Tout d'abord, nous avons constaté qu'il y a un grand nombre de petites fautes qui entament vraiment la valeur du texte grec (par exemple : p. 3, 1. 17 ËTous pour ËTous; p. 8, 1. 17 пa

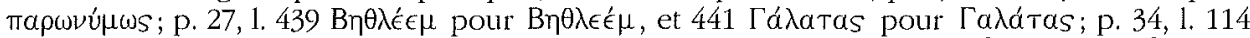

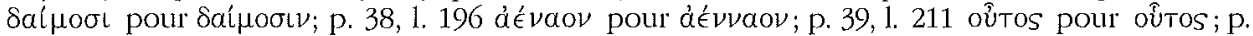

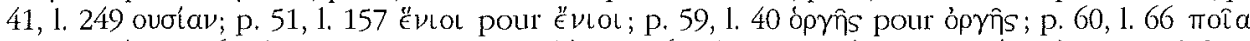

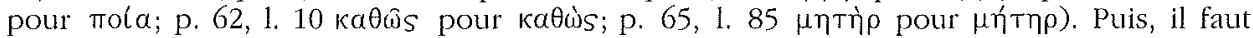
également déplorer que pas mal de références bibliques manquent dans l'apparat des sources (par exemple: p. 41, 1. 253: Act. 17, 28; p. 59, 1. 30-31: Lc. 18, 27; p. 65, 1. 71: Lc. 10, 19; p. 67, 1. 114-115: Mt. 10, 21).

En guise de conclusion de notre compte rendu, nous signalons un témoin resté inaperçu jusqu'ici. Il s'agit de l'Athous, Iviron 386 dont nous publierons une description très détaillée dans un livre à parâtre consacré à la tradition manuscrite de Maxime le Confesseur, théologien byzantin du $v{ }^{\mathrm{e}}$ siècle. Le manuscrit semble dater de la première partie du $x{ }^{e}{ }^{e}$ siècle; aucune note ne nous renseigne ni sur l'endroit de confection du manuscrit, ni sur son histoire postérieure; le volume, sauf pour les folios initiaux contenant un pinax et pour deux folios non numérotés qu'on trouve entre les actuels folios 125 et 127, revient à un seul scribe resté anonyme. La plus grande partie, bien connue des érudits, est consacrée à la querelle hésychaste. Mais il y a d'autres sections. Ainsi, les f. 169$193^{\mathrm{V}}$ contiennent une collection assez disparate de petits textes. Le texte qui nous intéresse ici est précédé immédiatement d'un petit florilège, qui se compose principalement de fragments pseudo-athanasiens (f. 170-174), et est suivi de la collection athonite des proverbes de Zenobius (f. $176^{\mathrm{V}}-190^{\mathrm{V}}$ ).

Ce sont donc les f. $174^{\mathrm{V}}-176$ qui contiennent une partie de la $\Theta \epsilon o \sigma o \phi i a$; nous en avons fait la collation, et voici nos constatations. Nous avons comparé le texte du manuscrit de la Sainte Montagne avec l'édition de Erbse; en effet, il est plus facile d'utiliser cette édition, car celle-ci donne l'édition de toutes les recensions de l'ceuvre.

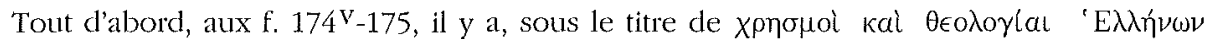
$\phi(\lambda o \sigma o ́ \phi \omega \nu$, une partie de la recension $\chi$. En voici l'inventaire: $\chi 9$ (le titre de ce fragment 


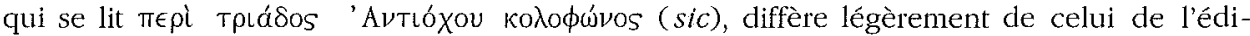
tion imprimée; on notera 7 autres variantes vis-à-vis du texte imprimé: 1. 226 voєpútepos]

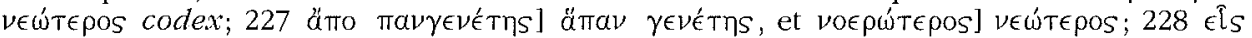

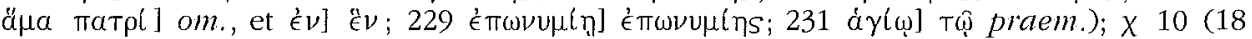

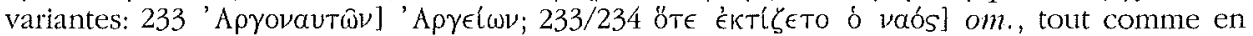

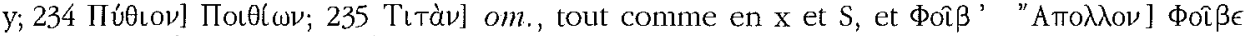

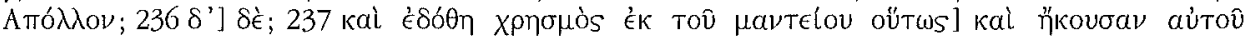

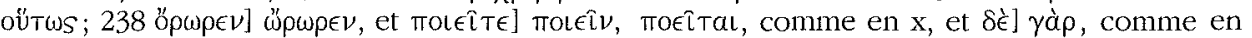

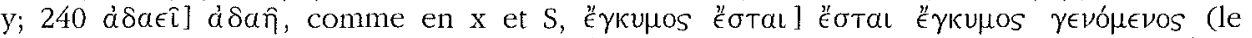

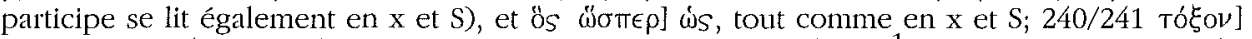

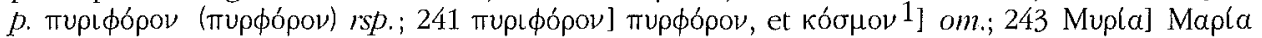

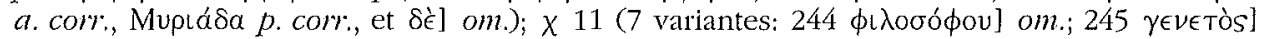

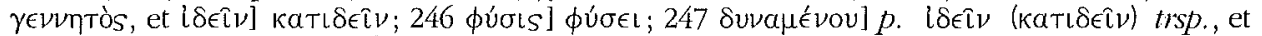

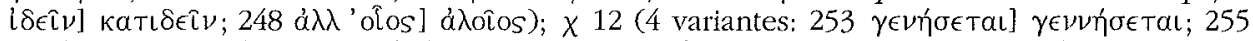

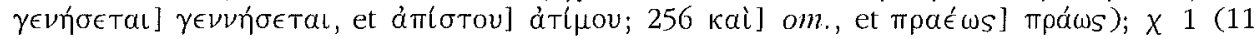

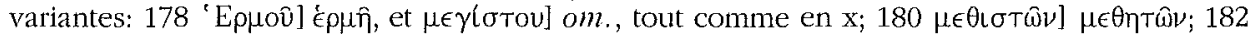

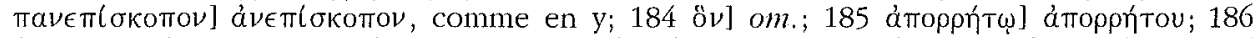

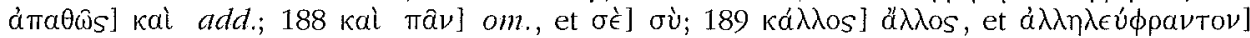

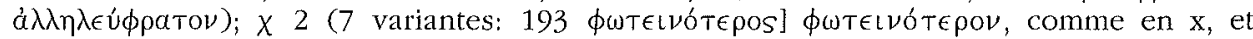

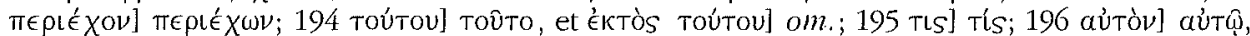

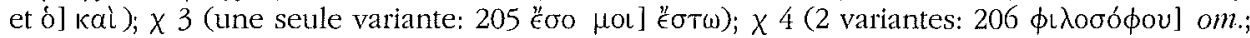

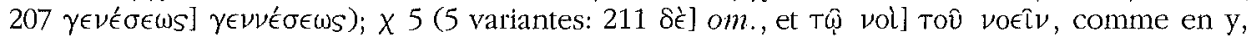

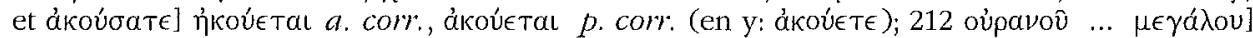

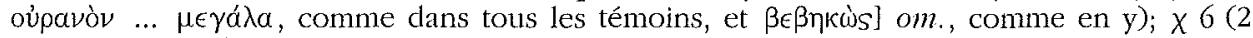

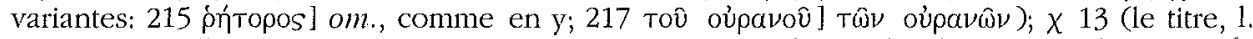

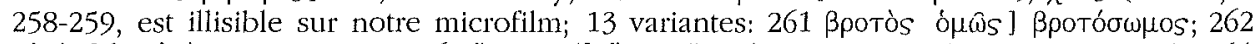

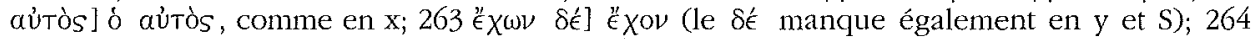

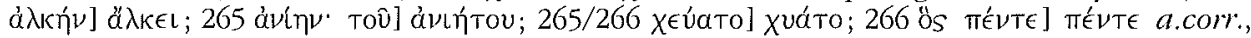

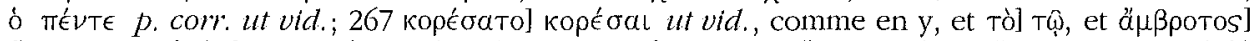

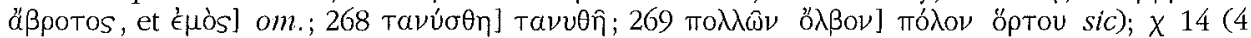

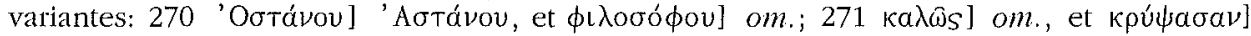

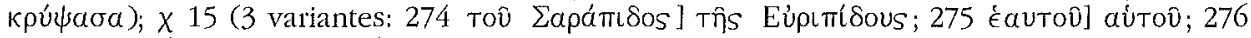

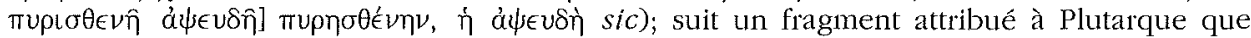
nous n'avons pas pu trouver tel quel, bien que ces mots semblent s'inspirer de la

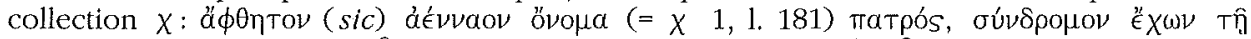

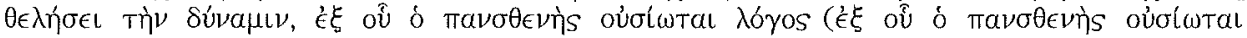

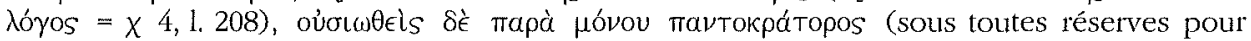
les deux derniers mots); $\chi 7$ (une seule variante: $218 \times(\lambda \omega \nu$ os $] X \in[\lambda \omega \nu$ ) $) ; \times 8$ (aucune variante).

La place que l'Atbous occupe dans le stemma de la collection $\chi$ (voir Erbse, Fragmenta, p. xxvi), reste un problème; en effet, l'Atbous suit tantôt la grande famille y, tantôt l'autre branche de la tradition, $x$; s'agit-il ici d'un témoin contaminé ?

Au f. $175^{\mathrm{V}}$, on trouve une combinaison de deux oracles d'Apollon, qui proviennent de la Passion de saint Artémios et sont attestés dans un bon nombre d'autres sources (pour la combinaison, voir le répertoire de la Bibliotheca bagiographica graeca 174e): un oracle

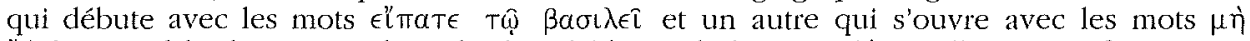
ő $\phi \epsilon \lambda \epsilon S$; seul le dernier oracle se lit dans l'édition de la $\Theta \epsilon o \sigma o \phi i ́ a:$ collection $\pi$, fragment 8 (notre manuscrit contient les 48-56 de $\pi$ 8); pour une étude de ces deux oracles, consulter H. Erbse, Fragmente, p. 129-134 et 153-155, ainsi que l'édition de H. Erbse, Fragmenta, p. xurv-Xuv; l'oracle $\epsilon^{\prime \prime}$ trate a été étudié dans deux articles qui portent le même titre

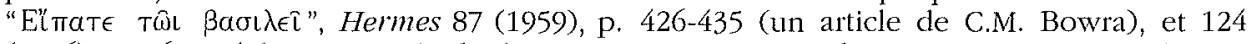
(1996), p. 367-374 (une petite étude de G. Fatouros). En voici les variantes qui caractérisent

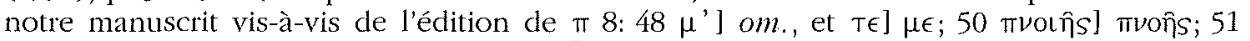

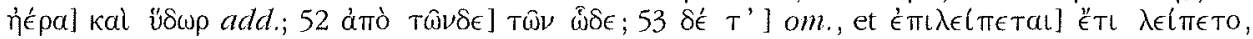

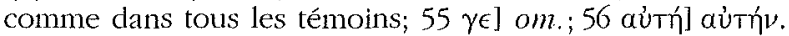

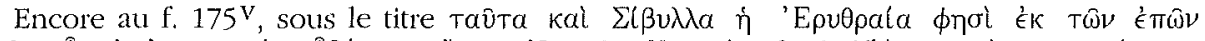

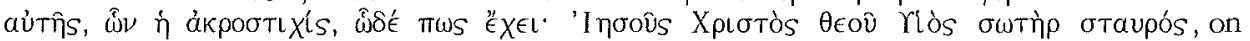
lit une partie des Oracula Sibyllina: VIII, 217 ('I $\delta \rho \omega \sigma \in t)$ - $250(\hat{n} \mu \omega \nu)$ dans l'édition de 
J. Geffcken, Die Oracula Sibyllina (Die griechischen christlichen Scbriftsteller der ersten drei Jabrbunderte), Leipzig, 1902, p. 153-157; on notera que ce fragment ne se lit ni dans l'édition de H. Erbse, ni dans celle de P.F. Beatrice, bien qu'une grande section y soit consacrée aux oracles des Sibylles; toutefois, H. Erbse, Fragmente, p. 97 n. 188, et p. 188, dit que ce fragment, que nous avons également découvert dans l'Atheniensis, Metochion Panagiou Tapbou $363, f .136^{\mathrm{V}}$, accompagne souvent ce qui suit dans notte manuscrit. En

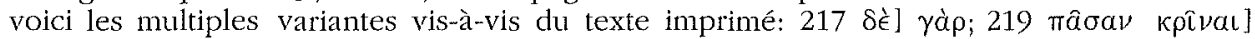

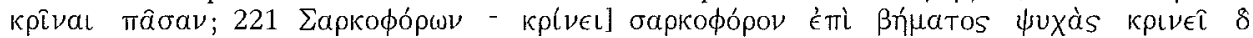

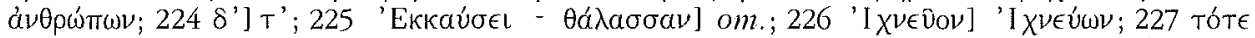

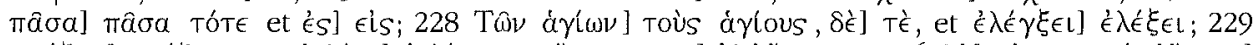

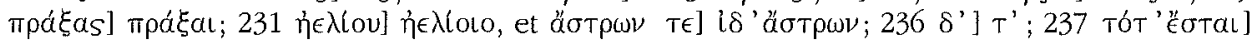

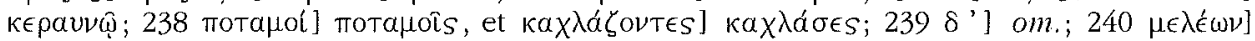

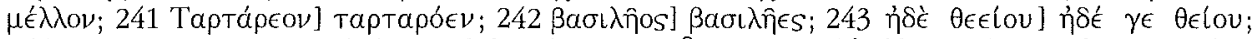

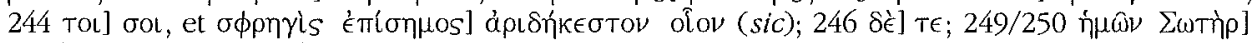

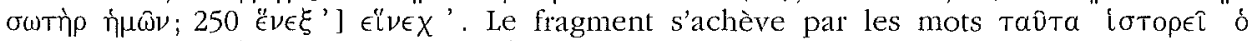

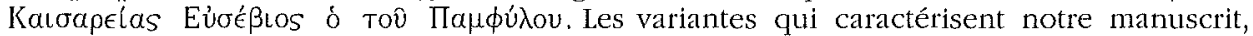
montrent que l'Athous se rapproche de la version de l'oracle telle qu'elle est conservée dans l'Oratio Constantini imperatoris ad sanctorum coetum d'Eusèbe de Césarée (Clavis Patrum Graecorum 3497); voir l'édition d'I.A. Heikel, Eusebius Werke, I, Über das Leben Constantins. Constantins Rede an die beilige Versammlung. Tricennatsrede an Constantin. (Die griechischen christlichen Schriftsteller der ersten drei Jabrbunderte), Leipzig, 1902, \$ XVIII (p. 179-181).

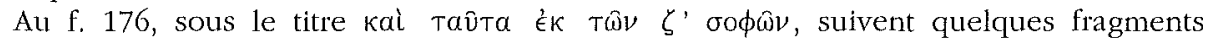
appartenant à la collection $\pi$ de la $\Theta \epsilon o \gamma \nu \omega o t a$; en voici l'inventaire détaillé: $\pi 7$ (2 vari-

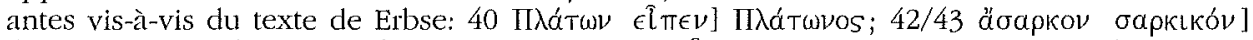

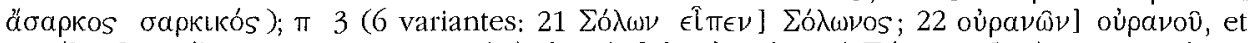

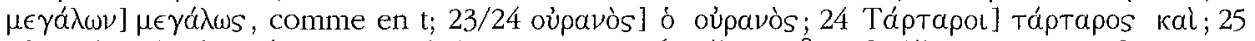

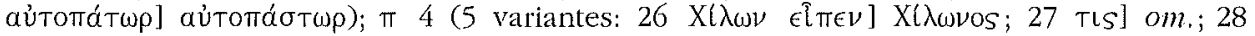

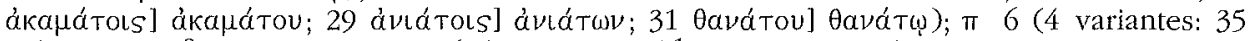

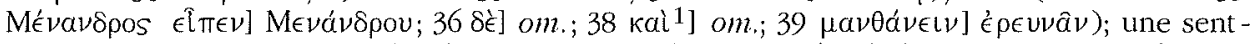

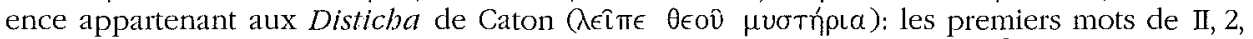
dans la traduction de Maxime Planude $\left({ }^{1}\right) ; \pi 2$ (2 variantes: 14 Blas $\epsilon[\pi \epsilon \nu]$ Bíavtos; 16

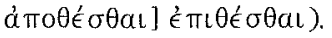

Pour la collection $\pi, l^{\prime}$ Atbous est clairement apparenté à la famille $t$ (voir Erbse, Fragmenta, p. xxx, stemma).

Toujours au f. 176, on lit encore quelques sections faisant partie de la collection w:

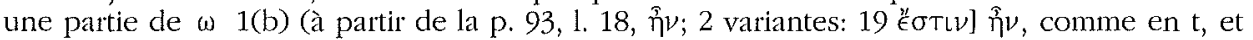

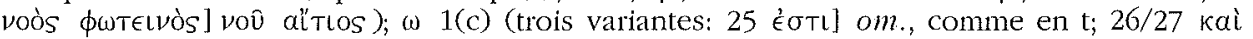

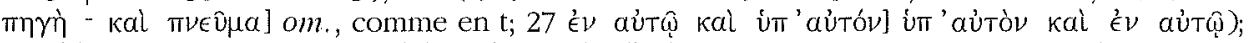
$\omega$ 1(e) (aucune variante); le début de $\omega$ 1(f) (le bas de la page étant découpé, le texte de ce dernier fragment est presque illisible; le dernier mot qu'on puisse y lire est $\pi \rho \hat{T} T \mathrm{v}$, au

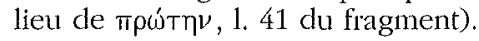

Pour la collection $\omega$, l'Athous est clairement apparenté à la famille t (pour la désignation $t$ utilisée pour la recension w, voir Erbse, Fragmenta, p. 92).

\section{(Katholieke Universiteit Leuven)}

Ricardo Di Donato, Hierà. Prolegomena ad uno studio storico antbropologico della religione greca, Università di Pisa, Edizioni Plus, 2001. 1 vol. $17 \times 24 \mathrm{~cm}, 368 \mathrm{p}$. (Didattica e ricerca. Manuali). ISBN : 88-8492-091-4.

Voici un manuel résolument peu conventionnel. Plutôt que d'offrir le panorama habituel de «tutte le verità di cui era necessaria la conoscenza » (p. 13), l'A. se place dans une perspective essentiellement historiographique et problématique. Nourri de l'approche « anthropologique » française de M. Mauss, L. Gernet et J.-P. Vernant, cet ouvrage invite

1 Voir l'édition de V. Ontoleva, Maximus Planudes. Disticha Catonis in Graecum translata, Roma, 1992 (Bibliotbeca Atena, 28), p. 8. 